\title{
Automated Breast Cancer Detection Models Based on Transfer Learning
}

\author{
Madallah Alruwaili * and Walaa Gouda
}

Citation: Alruwaili, M.; Gouda, W. Automated Breast Cancer Detection Models Based on Transfer Learning. Sensors 2022, 22, 876. https:/ / doi.org/10.3390/s22030876

Academic Editor: Steve Ling

Received: 30 November 2021

Accepted: 19 January 2022

Published: 24 January 2022

Publisher's Note: MDPI stays neutral with regard to jurisdictional claims in published maps and institutional affiliations.

Copyright: (C) 2022 by the authors. Licensee MDPI, Basel, Switzerland. This article is an open access article distributed under the terms and conditions of the Creative Commons Attribution (CC BY) license (https:// creativecommons.org/licenses/by/ $4.0 /)$.
College of Computer and Information Sciences, Jouf University, Sakaka 72341, Al Jouf, Saudi Arabia; wgaly@ju.edu.sa

* Correspondence: madallah@ju.edu.sa

\begin{abstract}
Breast cancer is among the leading causes of mortality for females across the planet. It is essential for the well-being of women to develop early detection and diagnosis techniques. In mammography, focus has contributed to the use of deep learning (DL) models, which have been utilized by radiologists to enhance the needed processes to overcome the shortcomings of human observers. The transfer learning method is being used to distinguish malignant and benign breast cancer by fine-tuning multiple pre-trained models. In this study, we introduce a framework focused on the principle of transfer learning. In addition, a mixture of augmentation strategies were used to prevent overfitting and produce stable outcomes by increasing the number of mammographic images; including several rotation combinations, scaling, and shifting. On the Mammographic Image Analysis Society (MIAS) dataset, the proposed system was evaluated and achieved an accuracy of $89.5 \%$ using (residual network-50) ResNet50, and achieved an accuracy of 70\% using the Nasnet-Mobile network. The proposed system demonstrated that pre-trained classification networks are significantly more effective and efficient, making them more acceptable for medical imaging, particularly for small training datasets.
\end{abstract}

Keywords: mammogram; breast cancer; deep learning; ResNet; Nasnet-Mobile; transfer learning; medical imaging

\section{Introduction}

Breast cancer is one of the most commonly diagnosed chronic illnesses in women. In 2012, there were approximately 1.17 million new cases globally, which represents one-fourth of all the new cases of cancer in women [1]. It is considered to be the second most diagnosed cancer in women, with the second most cancer deaths all over the world [2]. It can be treated by early discovery, which can significantly decrease breast cancer mortality. Breast cancer screening, mammography, is among the most effective ways to detect cancer at an early stage. Mammography is a low-dose X-ray diagnostic procedure for examining the internals of the breast, and it is currently the recommended approach for screening [3,4]. Despite the fact that mammography has enhanced the screening examinations sensitivities, particularly in dense breasts, the rate of false negatives (FNs) remains significant, owing to the presence of dense tissue that might obscure lesions. Other imaging modalities were considered, including ultrasound, magnetic resonance imaging (MRI), and infrared thermal imaging [5,6].

Masses and calcifications are the most common breast irregularities that may signify breast cancer. In preoperative evaluations of lesion extent and post-treatment surveillance in breast cancer patients, the identification and characterization of masses or calcifications are significant. In the mammogram, masses appear as bright regions of various sizes, margins (micro-lobular, circumscribed, indistinct, spiculated, and obscured), shapes (oval, irregular, round, and lobular), intensities, and contrasts of gray-level that rely on the tissues around them. These masses are called tumors and can be either "malignant", cancerous, or 
"benign", non-cancerous [3]. Calcifications, on the other hand, are small deposits of calcium that appear on mammograms as bright white splotches or spots on the background of the breasts' soft tissue. Usually, calcifications do not show up on ultrasounds, or on breast MRI images they never show up, while a common finding on mammograms is calcification $[7,8]$.

Several parameters, including poor image quality, the subtle nature of radiologists observations, eye exhaustion, or failure, can lead to missed detection. This leads to the task of automated mass detection and classification for both radiologists and computer-aided diagnosis (CAD) systems difficult. The fundamental problem in this case is the lack of a single method that provides satisfactory results for all images [9]. CAD systems have been built using a variety of machine learning techniques to improve the diagnostics performance of medical imaging for breast cancer. To address the machine learning difficulty, these methods are mainly based on classical classifiers that rely on hand-crafted features. As a result, these procedures are generally complicated, time-consuming, and require the involvement of specialists, particularly in the selection and extraction of characteristics. DL has shown promising results in resolving this problem recently. DL efficiency, however, is dependent on training with large, annotated datasets, which unfortunately, public mammography datasets lack $[9,10]$.

Transfer learning is a DL technique employed in this study that can help with the development of effective appropriate classifiers by transferring information from another area with big databases. A literature review is conducted in this study to identify currently utilized pre-trained models, as well as models that must be field-tested. Then we examined transfer learning (modified ResNet50, (MOD-RES), and Nasnet-Mobile) to see how accurate it was in classifying benign and malignant breast mammography abnormalities. Images from MIAS's public dataset are used in our proposed system's training and testing. The Nasnet-Mobile network has not yet been utilized, according to our research findings. MOD-RES, which outperforms state-of-the-art approaches, is also trained as a comparison.

Throughout this work, authors proposed a crossbred deep learning system for breast cancer classification and prognosis that uses two unique deep learning approaches to accurately detect early breast cancer symptoms from mammographic images. The proposed system has two significant phases: preprocessing, and classification. The preprocessing phase is used to improve the overall contrast of the image in order to make the images more visually appealed. The image is also resized and normalized to suit the size of the training model throughout that process. The classification stage, on the other hand, involves a variety of classifiers, and the most effective classifiers are chosen based on the classification error for each case.

The following are the key contributions of this research:

1. Using various evaluation metrics such as accuracy, precision, recall (sensitivity), specificity, and F1-Score. Extensive comparative comparisons were performed to assess the effectiveness of the proposed systems;

2. Mammograms show radiological indications that are readily detectable symptoms. As a result, deep learning-based methods can be used to automatically analyze mammograms, which significantly reduces analysis time;

3. To fine-tune the weights of pre-trained networks on small datasets, as well as train the weights of networks on large datasets, a customized version of ResNet50 (MOD-RES) and a hybrid version of Nasnet and Mobile net were utilized;

4. To improve the generalization effectiveness of the suggested method and prevent overfitting, a different training protocol assisted by different combinations of training policies (e.g., validation patience, and data augmentation) was used.

The remainder of the paper is laid out as follows: In Section 2, a literature review on the use of transfer learning to the classification of mammography abnormalities is conducted. The suggested experimental system, dataset, and model are provided in Section 3. In Section 4 , the results of the experiments are provided. The conclusion of the paper is included in Section 5. 


\section{Related Works}

Over the years, there have been numerous attempts to develop an automated methodology for identifying breast cancers from mammographic images. Several authors have employed typical machine learning methodologies, which include preprocessing images, feature extraction, feature selection to reduce the features size, and finally a classification algorithm to achieve the expected result. Transfer learning and Convolutional Neural Network (CNN) models, which are the most effective DL techniques currently in the medical domain, are proven to be superior to traditional methods [11,12]. In current history, DL has indeed been successfully implemented in the field of medicine with impressive outcomes and outstanding performance in different challenges compared with human activity. Various medical imaging systems using transfer learning techniques have also been developed to assist physicians and specialists in effective mammograms diagnosis, care, and follow-up examination $[13,14]$.

For example, Z. Hussain et al. [15] introduced how to work around CNNs and transfer learning networks to identify pre-segmented breast abnormalities in mammograms as benign or malignant, using a fusion of transfer learning visual geometry group VGG-16-16 (VGG-16) and data augmentation methods to address the tiny training data obtained from the Digital Mammography Screening Database (DDSM), achieving an accuracy of $88 \%$. Another approach presented by M. Alkhaleefah et al. [10], based on the double-shot transfer learning (DSTL) method, was used to enhance the total performance and accuracy of breast cancer classification pre-trained networks. DSTL uses a large dataset that is similar to the target dataset to fine-tune the learnable parameters (weights and biases) of the pre-trained network. The target dataset is then used to fine-tune the networks.

On the other hand, A. Perre et al. [16] proposed a transfer learning approach using three separate networks (VGG-f, VGG-m and caffe). During the fine-tuning process, the output of these pre-trained CNNs was examined twice; one with image normalization and the other without image normalization to identify abnormalities in mammograms. They tested the output of a support vector machine (SVM) fed with CNN extracted features and the combined the use of feature selection to enhance the $\mathrm{CNN}$ feature extraction. Another research presented by A. Khamparial et al. [17] implemented a modified version of VGG (MVGG), residual network, and mobile network. Research results using DDSM dataset demonstrated that the proposed learning model for hybrid transfers (fusion ImageNet and MVGG16) obtained an accuracy of $88.3 \%$, where the epoch numbers equal 15 . Just the modified VGG-16 design, on the other hand (MVGG16) provided $80.8 \%$ precision, and MobileNet provided $77.2 \%$ precision.

Furthermore, L. Falconi et al. [9] introduced a model including: VGG, ResNet, Xception, and Resnext. Their findings showed that in the CBIS-DDSM dataset, fine-tuning achieved the best classifier efficiency in VGG16 with an AUC value of 0.844 . While P. Kaur et al. [18] proposed a new technique, applied to the 322 image Mini-MIAS dataset. A pre-processing framework and integrated feature extraction using K-mean convergence for Speed-Up Robust Features (SURFs) are included. Even during the classification process, a new layer was introduced, which achieved $70 \%$ training ratios to $30 \%$ Deep Neural Network (DNN) and Multiclass SVM Tests. The outcome demonstrates that the consistency test using K-mean clustering and SVM of the suggested automatic DL approach is better than using a decision tree model. Another approach presented by K. Shaikh [19], which evaluates a CNN model using different datasets (MIAS, DDSM, BancoWeb, and LAPIMO) achieved accuracy equal to $87.5 \%$.

Another implementation presented by Wahab et al. [20] used a pre-trained CNN and applied its learnt parameters to another CNN to classify mitoses. Their proposed method attained precision, recall, and F-measure values of $0.50,0.80$, and 0.621 , respectively. Using the film mammography number 3 (BCDR-F03) dataset, Jiang et al. [21] achieved an accuracy of 0.88 using GoogleNet and 0.83 using AlexNet. On the other hand, Cao et al. [22] employed random forest dissimilarity to combine distinct feature groups without any finetuning on the source network layers (ResNet125). The dataset "ICIAR 2018" was employed, 
and classification accuracy was improved to $82.90 \%$. Furthermore, Charan et al. [23] trained a CNN with six convolution layers, four average-pooling layers, three fully connected (FC) layers, and a Softmax (SM) function on a $224 \times 224$ pixel input picture. The total accuracy of this network was $65 \%$ employing MIAS database.

The work presented by Charan et al. [23] is strikingly similar to ours in that it primarily trained and evaluated the model using images from the MIAS dataset. The proposed system demonstrates remarkable results that are more accurate than existing methods. Furthermore, compared with other models such as ResNet, VGG16, or DenseNet, the proposed improved ResNet50 system is lightweight. In terms of accuracy, our proposed system outperformed existing methods.

\section{Proposed System}

Figure 1 depicts the schematic methodology for the breast cancer detection system, which requires retraining transfer DL approaches (MOD-RES, and Nasnet-Mobile) over pre-processed images in the image datastore to learn discriminative and useful feature representations. At the beginning, the used datastore is described briefly, after that the proposed system's implementation specifics are discussed; including the proposed preprocessing algorithms, the main design, and the adopted approach's training methodology.

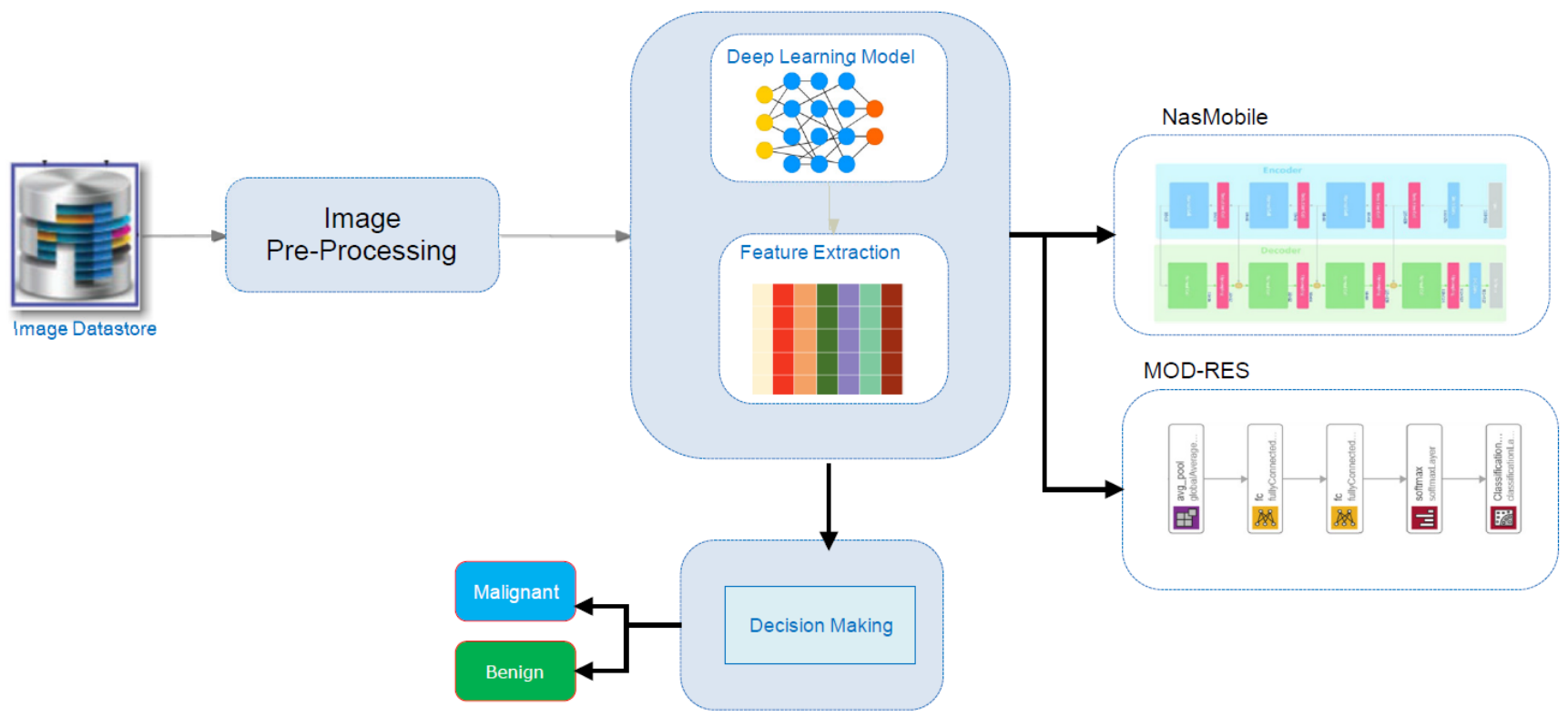

Figure 1. Schematic overview of the proposed system.

\subsection{MIAS Datasets}

In this work, MIAS was used. MIAS is an organization of scientific groups in the UK concerned with mammogram perception. A database of mammographic images collected through London's Royal Marsden Hospital by J. Suckling [24] has been developed. The archive includes 322 digitized films, and $2.3 \mathrm{~GB}$ of $8 \mathrm{~mm}$ are available. It also contains a report by radiologists on the tumor types and locations. The database is divided into seven sections, including healthy pairs of images and abnormal instances that included circumscribed masses, micro calcification, ill-defined masses, spiculated lesions, asymmetric densities, and architectural distortion. The overall number of images of malignant and benign mass cases are 51 and 48, respectively, before applying the augmentation methods.

\subsection{Image Pre-Processing}

This step includes data augmentation, image enhancement, image rescaling, and normalization, among other things. Since the model's network becomes more sophisticated, the number of parameters to learn increases as well, leading to overfitting. The process 
when a model learns the training data exceptionally well but fails to generalize effectively to subsequent testing data is known as overfitting. Overfitting is a prevalent issue in DL models, and the risk of coming into it increases when the training dataset is limited, as it was in this work. After that the MIAS dataset was divided into three mutually exclusive sets (e.g., preparing, verification, and evaluating sets) to overcome the overfitting issue created by the small number of training photos. The data augmentation was used to prevent skewed prediction outcomes. Augmented images with corresponding masks such as rotation, reflection, shifting, and scaling were generated for each image in the dataset.

The accuracy of a raw image produced by an electronic detector is simply inadequate, reducing the availability of detection and diagnosis. To improve the quality of mammographic images, image enhancement techniques should be used. Furthermore, training DNNs on top of preprocessed images rather than raw image data will significantly reduce the DNNs' generalization error and training time. As a result, an appropriate image enhancement technique was proposed to improve the low quality of the images before feeding them into the proposed system. First, the image's small details, textures, and low contrast were improved using adaptive contrast enhancement based on redistribution of the input image's lightness values, as shown in Figure 2. Consequently, this approach will improve the visibility of the edges and curves in each part of an image while also enhancing the image's local contrast.

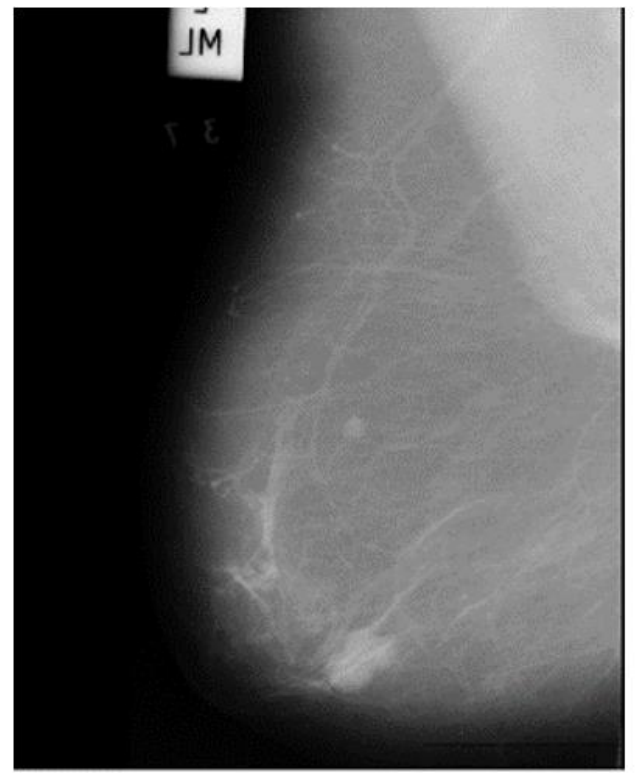

(a)

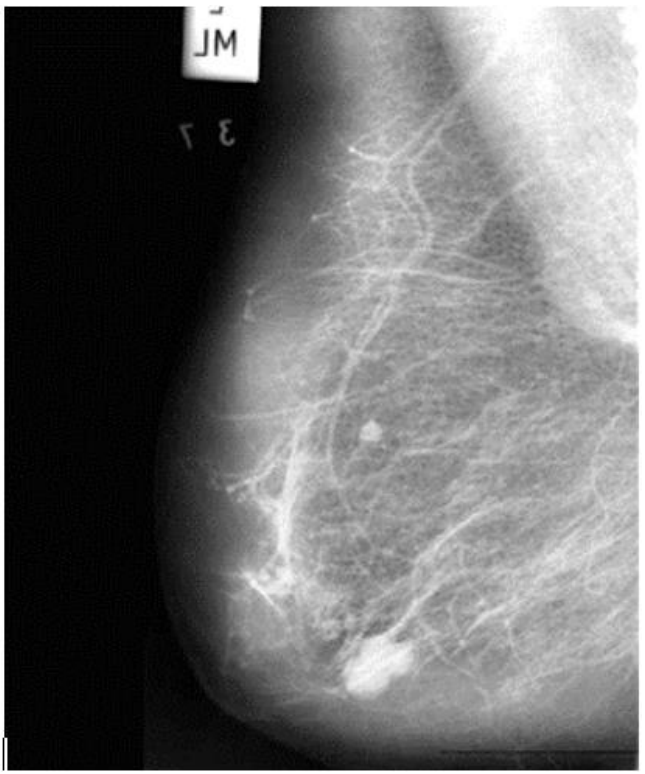

(b)

Figure 2. Output of proposed image enhancement process, (a) raw image; (b) enhanced image.

Since the images in the dataset are grayscale, the images must replicate three times to obtain an RGB image. We consider it is appropriate to normalize the image to a range of values of 256 gray levels, so that the pixels intensity of all images is normalized between -1 and 1 to ensure that the data are within specific ranges and noise is removed. Normalization has the benefit of ensuring the model is less vulnerable to slight variations in weights and making it easier to optimize.

\subsection{Proposed Learning Methods}

One of the most significant challenges researchers confront when analyzing medical data is the restricted number of available datasets. DL models frequently require a massive amount of data as well as expert data labeling, which are both expensive and time-consuming. The proposed system's main architecture is based on the transfer learning models. The massive number of structures and hyperparameters to be determined is the 
most difficult challenge when using DL models (e.g., learning rate, number of batch size, number of frozen layers, and number of epochs, etc.). The effects of various hyperparameters value on the performance of the proposed systems were investigated.

Transfer learning using Nasnet-Mobile, and a modified version of ResNet50 (named MOD-RES) were used to classify breast cancer X-ray images into two categories (Benign and Malignant). In addition, to address the lack of data, we used a transfer learning technique that involved oversampling by duplicating the number of images. Figure 1 shows a schematic representation of the proposed system for the prediction of benign and malignant tumors, including pre-trained Nasnet-Mobile, and MOD-RES models.

\subsubsection{Nasnet-Mobile}

Since AlexNet gained world attention, the development of the CNN has gone through three phases, the principles which are called Deeper is Better, Architecture Engineering, and AutoML. Nasnet is an extensible CNN model that stands for Neural Search Architecture (NAS) Network. The convolutional neural network Nasnet-Large was trained on over a million photos from the ImageNet collection [25]. The basic concepts differ from typical models such as GoogleNet, and it is expected to lead to a big breakthrough in AI in the near future. It is made up of fundamental building blocks that are tuned using reinforcement learning. A cell is made up of only a few functions and is repeated many times depending on the network's capacity requirements [26]. Nasnet-Mobile is a mobile version of Nasnet with 12 cells, 5.3 million parameters, and 564 million multiply accumulates [27]. This network has never been utilized before to classify mammographic images, as far as we know.

\subsubsection{MOD-RES}

In 2015, He K. et al. [28] developed ResNet50, a new a residual learning component to the CNN architecture. A standard layer with a skipped connection compensates the residual unit. The skip connection enables a layer's input signal to traverse the network by linking it to that layer's output. As a result of the residual units, an extremely deep 152-layer model was trained, which won the 2015 LSVRC2015 competition. Its innovative residual structure allows for a more straightforward gradient flow and more efficient training. It has a top-five error rate of less than 3.6 percent. ResNet has 34, 50, and 101 layers in other versions.

In this section, we go over the details of a potential solution based on a modified version of the ResNet50 [28] model, as shown in Figure 3. Figure 3a depicts the original ResNet50 model, while in Figure 3b, the latest layers are altered by adding one FC layer as well as replacing both the existing FC layer and Softmax layer to construct the proposed model. The ResNet50 model's original layers were pre-trained on the ImageNet dataset [25]. As a result, the additional layers will assign random weights firstly, after that the backpropagation technique, which is the basic algorithm for training neural network models, is used to update all model weights throughout training.

In the MOD-RES model, shown in Figure 3b, the first FC was replaced with a new FC layer with size 512 and one FC layer with size 2, number of classes, was added after the replaced FC layer and before the Softmax layer which also was replaced with new the Softmax layer. Based on what was mentioned by Basha, S.S et al. [29] when dealing with small datasets, the network needs more FC layers than when dealing with larger datasets. Any neuron from the previous layer is connected to every other neuron in the next layer in the FC layer, and each value contributes to predicting how well a value fits a given class. The output of the final FC layer is then redirected to an activation function, which calculates the class scores. One of DNN's most common classifiers is Softmax, which computes the probability distribution of the $n$ output groups through its equations. The only drawback for adding a single FC layer is that it is extremely computationally intensive. 


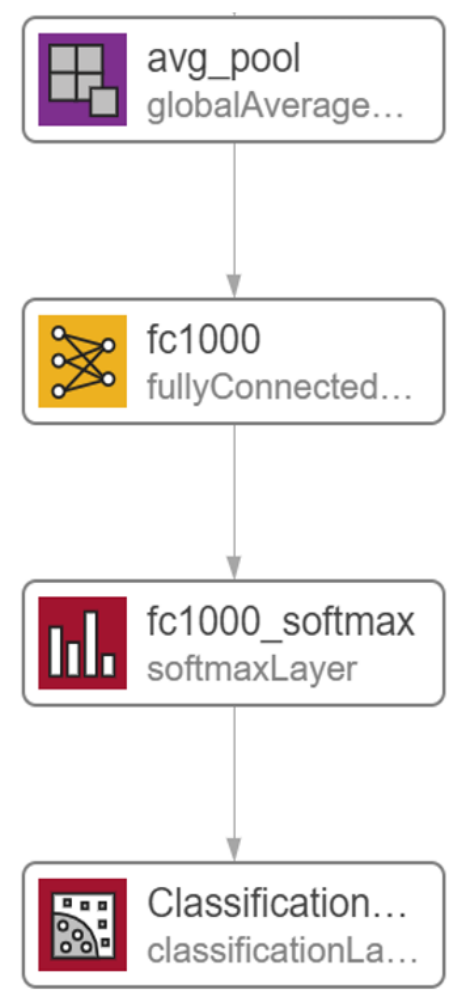

(a)

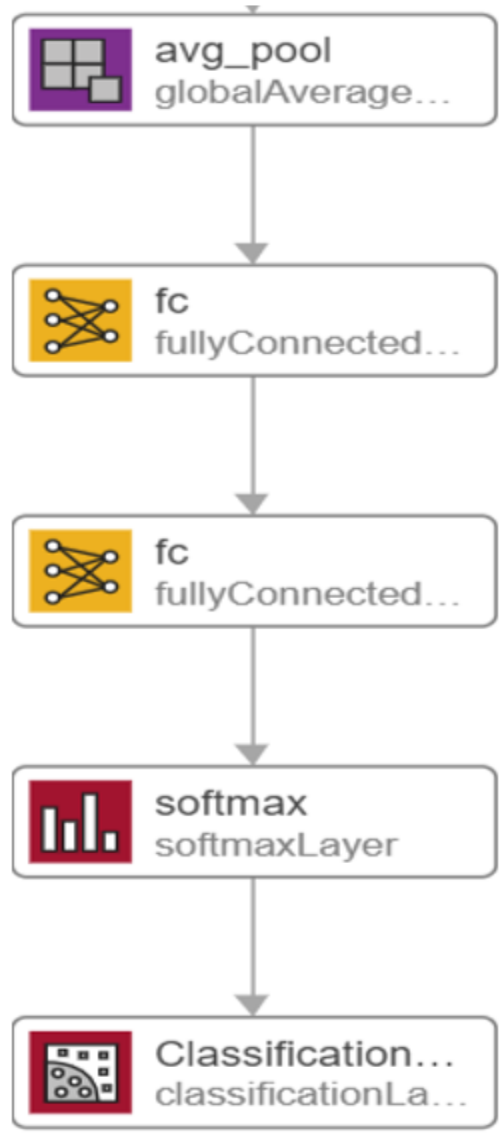

(b)

Figure 3. Modified version of proposed ResNet50 model; (a) original pre-trained model; (b) MOD-RES.

\section{Experimental Results}

Several sufficiently large experiments were performed on the MIAS dataset to demonstrate the efficiency of the proposed systems and to equate their results to the existing state-of-the-art approaches. The proposed system's code was written throughout MATLAB R2020b and evaluated on a Windows 10 machine with a Core i7-4650U CPU and 8 GB of RAM. All tests were carried out using an $80 \%$ random array of mammographic images as a training collection for the proposed DL systems, according to the proposed training scheme. During the learning process, $10 \%$ of the training data was chosen at random and used as a validation set to assess their abilities and save the weight combinations with the highest accuracy value.

The proposed system is pre-trained on the MIAS dataset using the Adam and sigmoid optimizer with a learning rate strategy that decreases the learning rate when learning becomes stagnant for a period (i.e., validation patience). The following hyperparameters were used for training either in the Adam or Sigmoid optimizers: number of epochs $=15$, batch size varying from 32 to 128 with a move of double its previous value; patience $=6$; and momentum $=0.95$. Finally, we incorporate a batch re-balancing strategy to enhance infection form distribution at the batch stage. 
The batch normalization was utilized because of its effectiveness in preventing network overfitting. Because several steps in such algorithms contain a degree of randomness, DNN methods always provide outcomes with a degree of variability [30]. As a result, ensemble learning is one technique to increase the performance of DNN algorithms. Throughout this study, we suggest that stacking generalization can be implemented through doing numerous training runs of the same model, which we named the multiple-runs ensemble.

\subsection{Assessment Metrices}

To evaluate performance, our proposed system is compared with other systems using the following performance metrics:

$$
\begin{gathered}
\text { Overall Accuracy }=\frac{\mathrm{TP}+\mathrm{TN}}{\mathrm{TP}+\mathrm{TN}+\mathrm{FP}+\mathrm{FN}} \\
\text { Precision }=\frac{\mathrm{TP}}{\mathrm{TP}+\mathrm{FP}} \\
\text { Sensetivity }=\text { Recall }=\frac{\mathrm{TP}}{\mathrm{TP}+\mathrm{FN}} \\
\mathrm{F} 1-\text { Score }=\frac{2 * \text { Precision } * \text { Recall }}{\text { Precision }+ \text { Recall }}
\end{gathered}
$$

Included are, true positives (TPs) (sufferers correctly defined as having malignant mass), true negatives (TNs) (sufferers correctly reported as having benign mass), false positives (FPs) (sufferers with benign mass identified as having malignant mass), and FN (sufferers with malignant mass identified as not having the disease).

\subsection{Results of the Proposed Systems}

In this section we report the different experiments results of the proposed systems using the MIAS dataset with $80-20 \%$ train-test split. That split is selected, to ensure that execution times were not prohibitive. In the first experiment, we trained the MOD-RES and Nasnet-Mobile models for 15 epochs using 10\% of the training set as a validation set, a batch size ranging from 32 up to 128, and a learning rate ranging from 0.0002 up to 0.0008 and freeze the weights of the first 50 layers of the model for MOD-RES model and the first 250 layers for Nasnet-Mobile model. We executed the training three times and monitored the average accuracy measures over the validation set. Tables $1-4$ show the average accuracy of an ensemble of the modified models. As mentioned previously, we built model the ensemble in a way of multiple runs (two runs) to train the same model with the same parameters. An observation that can be made is that the accuracy varies from run to run as the weights are initialized randomly each run, only the best run result is saved and shown in Figures 4-7. Comparing the two models, the best achieved accuracy for MOD-RES and Nasnet-Mobile models are 70\% for both models.

Table 1. Average accuracy for MOD-RES model with freeze first 50 layers, epochs $=15$, optimizer $=$ Adam .

\begin{tabular}{ccccccccc}
\hline \multirow{2}{*}{$\begin{array}{c}\text { Learning } \\
\text { Rate }\end{array}$} & \multicolumn{8}{c}{ Ensemble Using Several Runs } \\
\cline { 2 - 9 } & \multicolumn{1}{c}{ Batch Size $=\mathbf{3 2}$} & \multicolumn{1}{c}{ Batch Size = $\mathbf{6 4}$} & \multicolumn{2}{c}{ Batch Size = 128 } & \multicolumn{2}{c}{ Batch Size = 265 } \\
\cline { 2 - 9 } & $\mathbf{1}$ & $\mathbf{2}$ & $\mathbf{1}$ & $\mathbf{2}$ & $\mathbf{1}$ & $\mathbf{2}$ & $\mathbf{1}$ & $\mathbf{2}$ \\
\hline 0.0002 & 0.4 & 0.4 & 0.65 & 0.55 & 0.55 & 0.6 & 0.45 & 0.65 \\
0.0004 & 0.55 & 0.5 & 0.6 & 0.6 & 0.55 & 0.6 & 0.55 & 0.5 \\
0.0006 & 0.55 & 0.45 & 0.45 & 0.5 & 0.6 & 0.55 & 0.5 & 0.6 \\
0.0008 & 0.55 & 0.65 & 0.55 & 0.6 & 0.65 & 0.6 & 0.65 & 0.7 \\
\hline
\end{tabular}


Table 2. Average accuracy for MOD-RES model with freeze first 50 layers, epochs $=15$, optimizer $=$ Sigmoid .

\begin{tabular}{ccccccccc}
\hline \multirow{2}{*}{$\begin{array}{c}\text { Learning } \\
\text { Rate }\end{array}$} & \multicolumn{8}{c}{ Ensemble Using Several Runs } \\
\cline { 2 - 10 } & \multicolumn{1}{c}{ Batch Size = $\mathbf{3 2}$} & \multicolumn{1}{c}{ Batch Size = 64 } & \multicolumn{2}{c}{ Batch Size = 128 } & \multicolumn{2}{c}{ Batch Size = 265 } \\
\cline { 2 - 10 } & $\mathbf{1}$ & $\mathbf{2}$ & $\mathbf{1}$ & $\mathbf{2}$ & $\mathbf{1}$ & $\mathbf{2}$ & $\mathbf{1}$ & $\mathbf{2}$ \\
\hline 0.0002 & 0.45 & 0.4 & 0.7 & 0.5 & 0.6 & 0.5 & 0.55 & 0.65 \\
0.0004 & 0.5 & 0.5 & 0.55 & 0.45 & 0.55 & 0.55 & 0.55 & 0.55 \\
0.0006 & 0.55 & 0.5 & 0.6 & 0.5 & 0.55 & 0.55 & 0.65 & 0.5 \\
0.0008 & 0.45 & 0.55 & 0.55 & 0.6 & 0.65 & 0.55 & 0.5 & 0.45 \\
\hline
\end{tabular}

Table 3. Average accuracy for Nasnet-Mobile model with freeze first 250 layers, epochs $=15$, optimizer $=$ Adam .

\begin{tabular}{ccccccccc}
\hline \multirow{2}{*}{$\begin{array}{c}\text { Learning } \\
\text { Rate }\end{array}$} & \multicolumn{8}{c}{ Ensemble Using Several Runs } \\
\cline { 2 - 9 } & \multicolumn{1}{c}{ Batch Size $=\mathbf{3 2}$} & \multicolumn{1}{c}{ Batch Size $=\mathbf{6 4}$} & \multicolumn{2}{c}{ Batch Size = 128 } & \multicolumn{2}{c}{ Batch Size = 265 } \\
\cline { 2 - 9 } & $\mathbf{1}$ & $\mathbf{2}$ & $\mathbf{1}$ & $\mathbf{2}$ & $\mathbf{1}$ & $\mathbf{2}$ & $\mathbf{1}$ & $\mathbf{2}$ \\
\hline 0.0002 & 0.35 & 0.4 & 0.4 & 0.4 & 0.45 & 0.5 & 0.55 & 0.5 \\
0.0004 & 0.4 & 0.45 & 0.55 & 0.4 & 0.6 & 0.55 & 0.45 & 0.45 \\
0.0006 & 0.35 & 0.35 & 0.45 & 0.5 & 0.5 & 0.5 & 0.5 & 0.55 \\
0.0008 & 0.4 & 0.4 & 0.55 & 0.6 & 0.7 & 0.6 & 0.55 & 0.5 \\
\hline
\end{tabular}

Table 4. Average accuracy for Nasnet-Mobile model with freeze first 250 layers, epochs $=15$, optimizer $=$ Sigmoid.

\begin{tabular}{ccccccccc}
\hline \multirow{2}{*}{$\begin{array}{c}\text { Learning } \\
\text { Rate }\end{array}$} & \multicolumn{8}{c}{ Ensemble Using Several Runs } \\
\cline { 2 - 10 } & \multicolumn{1}{c}{ Batch Size $=\mathbf{3 2}$} & \multicolumn{1}{c}{ Batch Size $=\mathbf{6 4}$} & \multicolumn{2}{c}{ Batch Size = 128 } & \multicolumn{2}{c}{ Batch Size = 265 } \\
\cline { 2 - 10 } & $\mathbf{1}$ & $\mathbf{2}$ & $\mathbf{1}$ & $\mathbf{2}$ & $\mathbf{1}$ & $\mathbf{2}$ & $\mathbf{1}$ & $\mathbf{2}$ \\
\hline 0.0002 & 0.4 & 0.4 & 0.5 & 0.4 & 0.55 & 0.5 & 0.45 & 0.4 \\
0.0004 & 0.35 & 0.5 & 0.35 & 0.35 & 0.45 & 0.5 & 0.4 & 0.4 \\
0.0006 & 0.4 & 0.45 & 0.5 & 0.45 & 0.4 & 0.4 & 0.5 & 0.45 \\
0.0008 & 0.35 & 0.35 & 0.45 & 0.4 & 0.5 & 0.45 & 0.4 & 0.5 \\
\hline
\end{tabular}

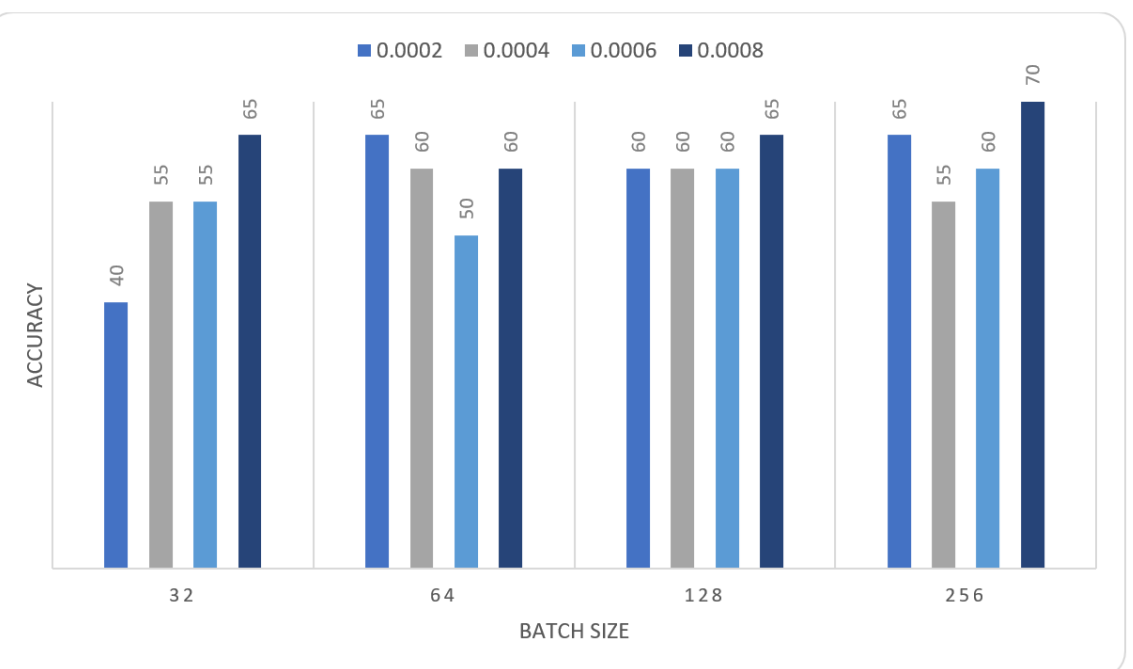

Figure 4. Average accuracy for MOD-RES model with freeze first 50 layers, epochs $=15$, optimizer $=$ Adam . 


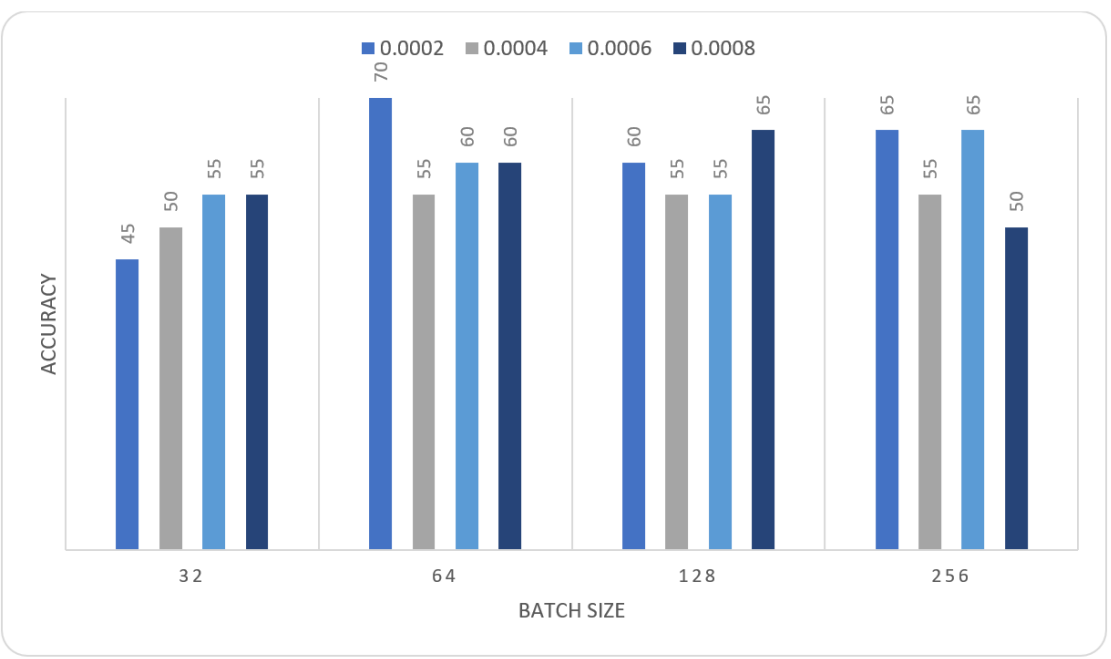

Figure 5. Average accuracy for MOD-RES model with freeze first 50 layers, epochs $=15$, optimizer $=$ Sigmoid.

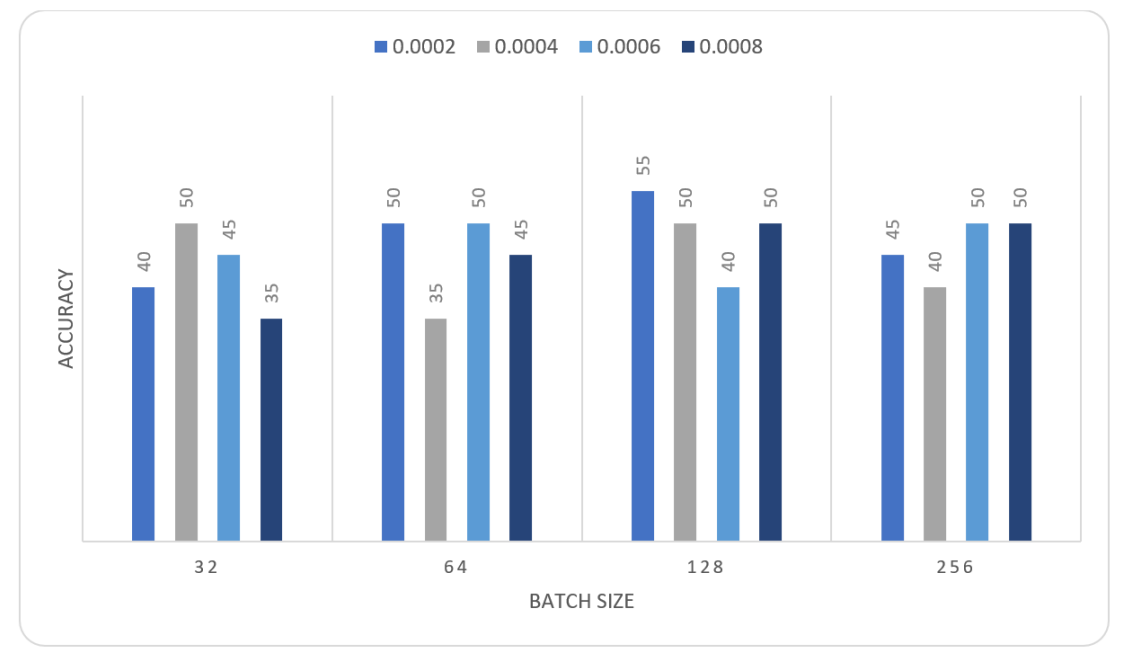

Figure 6. Average accuracy for Nasnet-Mobile model with freeze first 50 layers, epochs $=15$, optimizer $=$ Adam.

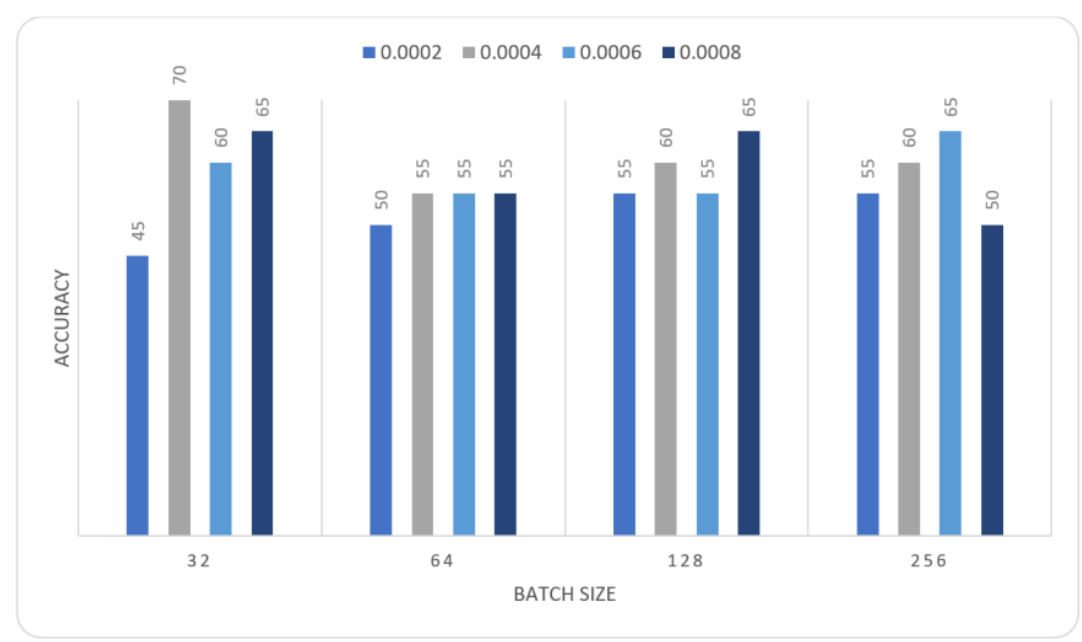

Figure 7. Average accuracy for Nasnet-Mobile model with freeze first 250 layers, epochs $=15$, optimizer $=$ Sigmoid 
The obtained results were insufficient; therefore, we devised a new strategy to address the issue: we performed oversampling by duplicating images once before applying augmentation to the images, and then MOD-RES was applied. So that in the second experiment, the MOD-RES model was trained only for 15 epochs using $10 \%$ of the training set as a validation set, a batch size of 32, 64, 128, and 265, and a learning rate ranging from 0.0002 up to 0.0008 with freezing the weights of the first 50 layers. The training was executed two times and monitored the average accuracy measures over the validation set. Tables 5 and 6 show the average accuracy of an ensemble of the MOD-RES model which is $89.5 \%$ and $86.8 \%$ using the Adam and Sigmoid optimizer, respectively.

Table 5. Average accuracy for MOD-RES model with freeze first 50 layers, epochs = 15, optimizer $=$ Adam .

\begin{tabular}{ccccccccc}
\hline \multirow{2}{*}{$\begin{array}{c}\text { Learning } \\
\text { Rate }\end{array}$} & \multicolumn{8}{c}{ Ensemble Using Several Runs } \\
\cline { 2 - 9 } & \multicolumn{1}{c}{ Batch Size $=\mathbf{3 2}$} & \multicolumn{2}{c}{ Batch Size = $\mathbf{6 4}$} & \multicolumn{2}{c}{ Batch Size = 128 } & \multicolumn{2}{c}{ Batch Size = 265 } \\
\cline { 2 - 9 } & $\mathbf{1}$ & $\mathbf{2}$ & $\mathbf{1}$ & $\mathbf{2}$ & $\mathbf{1}$ & $\mathbf{2}$ & $\mathbf{1}$ & $\mathbf{2}$ \\
\hline 0.0002 & 0.737 & 0.711 & 0.658 & 0.658 & 0.553 & 0.605 & 0.526 & 0.711 \\
0.0004 & 0.5 & 0.789 & 0.658 & 0.711 & 0.763 & 0.737 & 0.632 & 0.763 \\
0.0006 & 0.395 & 0.605 & $\mathbf{0 . 8 9 5}$ & 0.842 & 0.816 & 0.711 & 0.658 & 0.658 \\
0.0008 & 0.553 & 0.526 & 0.711 & 0.684 & 0.526 & 0.789 & 0.579 & 0.763 \\
\hline
\end{tabular}

Table 6. Average accuracy for MOD-RES model with freeze first 50 layers, epochs $=15$, optimizer $=$ Sigmoid.

\begin{tabular}{ccccccccc}
\hline \multirow{2}{*}{$\begin{array}{c}\text { Learning } \\
\text { Rate }\end{array}$} & \multicolumn{8}{c}{ Ensemble Using Several Runs } \\
\cline { 2 - 9 } & \multicolumn{1}{c}{ Batch Size $=\mathbf{3 2}$} & \multicolumn{1}{c}{ Batch Size $=\mathbf{6 4}$} & \multicolumn{2}{c}{ Batch Size = 128 } & \multicolumn{2}{c}{ Batch Size = 265 } \\
\cline { 2 - 9 } & $\mathbf{1}$ & $\mathbf{2}$ & $\mathbf{1}$ & $\mathbf{2}$ & $\mathbf{1}$ & $\mathbf{2}$ & $\mathbf{1}$ & $\mathbf{2}$ \\
\hline 0.0002 & 0.632 & 0.711 & 0.684 & 0.789 & 0.816 & 0.737 & 0.526 & 0.5 \\
0.0004 & 0.5 & 0.526 & 0.632 & 0.789 & $\mathbf{0 . 8 6 8}$ & 0.737 & 0.711 & 0.5 \\
0.0006 & 0.605 & 0.526 & 0.789 & 0.658 & 0.684 & 0.737 & 0.579 & 0.474 \\
0.0008 & 0.658 & 0.763 & 0.605 & 0.737 & 0.658 & 0.632 & 0.579 & 0.526 \\
\hline
\end{tabular}

Figures 8 and 9 summarize the previous tables, which display the average accuracy of an ensemble of the MOD-RES model using the Adam optimizer, and Sigmoid optimizer for the best run. It can be noticed that the best result obtained using Adam and Sigmoid optimizers provide the result with accuracy equal to $89.5 \%$, and $86.8 \%$ respectively. These results revealed that using oversampling for the images improves the overall accuracy of the proposed model (MOD-RES).

Table 7 shows the best results obtained for using the MOD-RES model with and without oversampling, and the Nasnet-Mobile. The disparity in performance can be explained by the model's inability to learn the large number of parameters due to the small number of images.

Table 7. Best result for all models.

\begin{tabular}{cccc}
\hline $\begin{array}{c}\text { Quantitative } \\
\text { Measures }\end{array}$ & $\begin{array}{c}\text { MOD-RES Model } \\
\text { (Oversampling) }\end{array}$ & MOD-RES Model & $\begin{array}{c}\text { Nasnet-Mobile } \\
\text { Model }\end{array}$ \\
\hline Overall Accuracy & $\mathbf{8 9 . 5}$ & 70 & 70 \\
Precision & $\mathbf{8 9 . 5}$ & 64.3 & 83.3 \\
Recall & 89.5 & $\mathbf{9 0}$ & 50 \\
F1-score & $\mathbf{8 9 . 5}$ & 75 & 62.5 \\
\hline
\end{tabular}




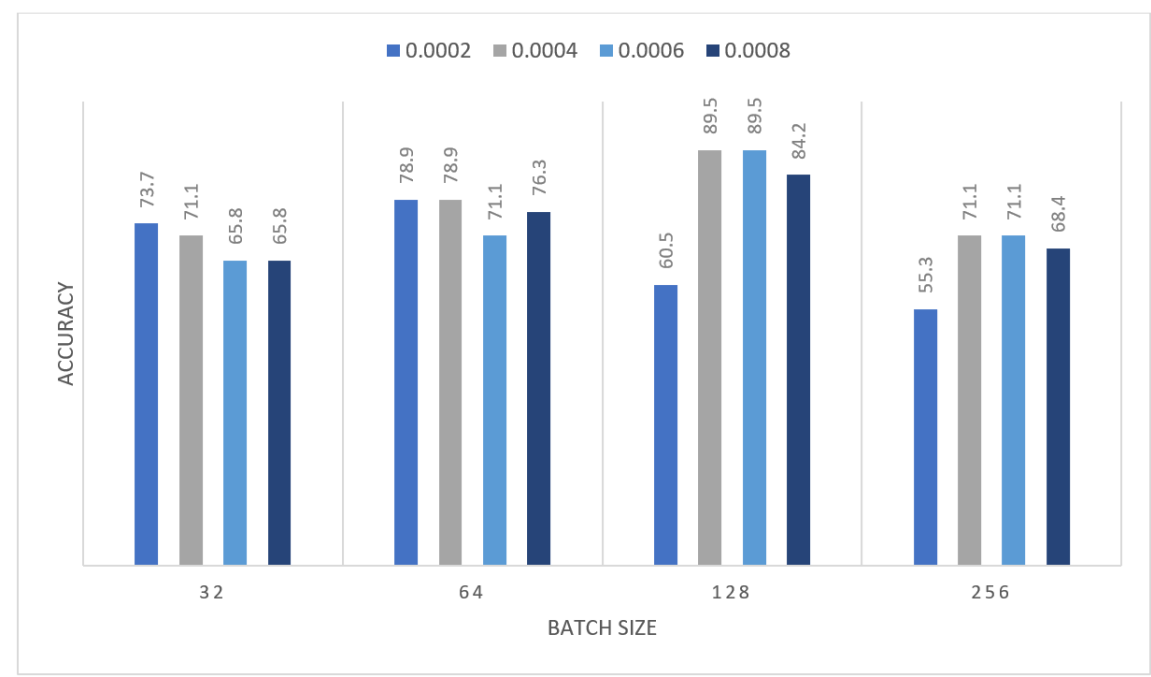

Figure 8. Average accuracy for MOD-RES model with freeze first 50 layers, epochs $=15$, optimizer $=$ Adam .

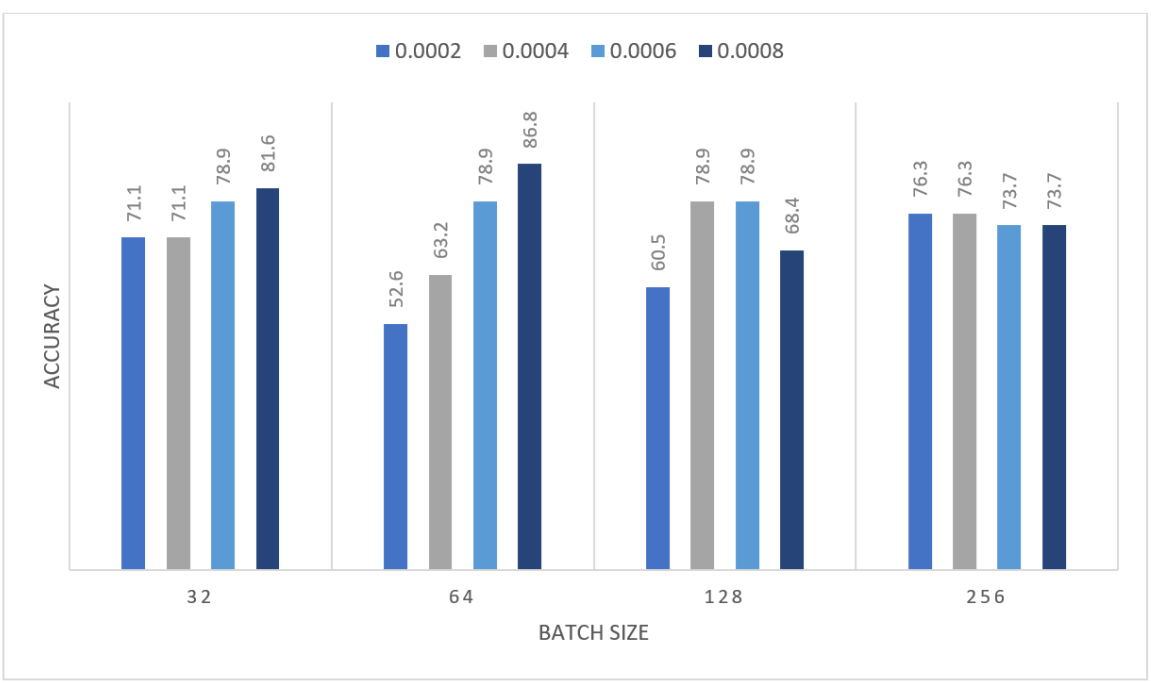

Figure 9. Average accuracy for MOD-RES model with freeze first 50 layers, epochs $=15$, optimizer $=$ Sigmoid.

Figure 10 shows that the oversampling + MOD-RES model is used in the efficiency comparison of the proposed system with current state-of-the-art approaches, in addition to its usefulness in leveraging the great strengths of each classifier. These findings bolstered the case for implementing the proposed system in real-world environments to help radiologists diagnose breast infection more accurately using mammograms while also reducing their workload.

\subsection{Comparison to State-of-the-Art Methods}

The proposed system's performance and reliability are compared with the most recent research in mammogram mass detection systems. In this section, we present the proposed system (oversampling + MOD-RES) outcomes and compare them to existing methods (see Table 8). As revealed in Table 8, the proposed system demonstrates remarkable results that are more accurate than existing methods. Furthermore, compared with other models such as VGG16 or DenseNet, the proposed improved ResNet50 system is lightweight. In terms of accuracy, our proposed system outperformed existing methods. 


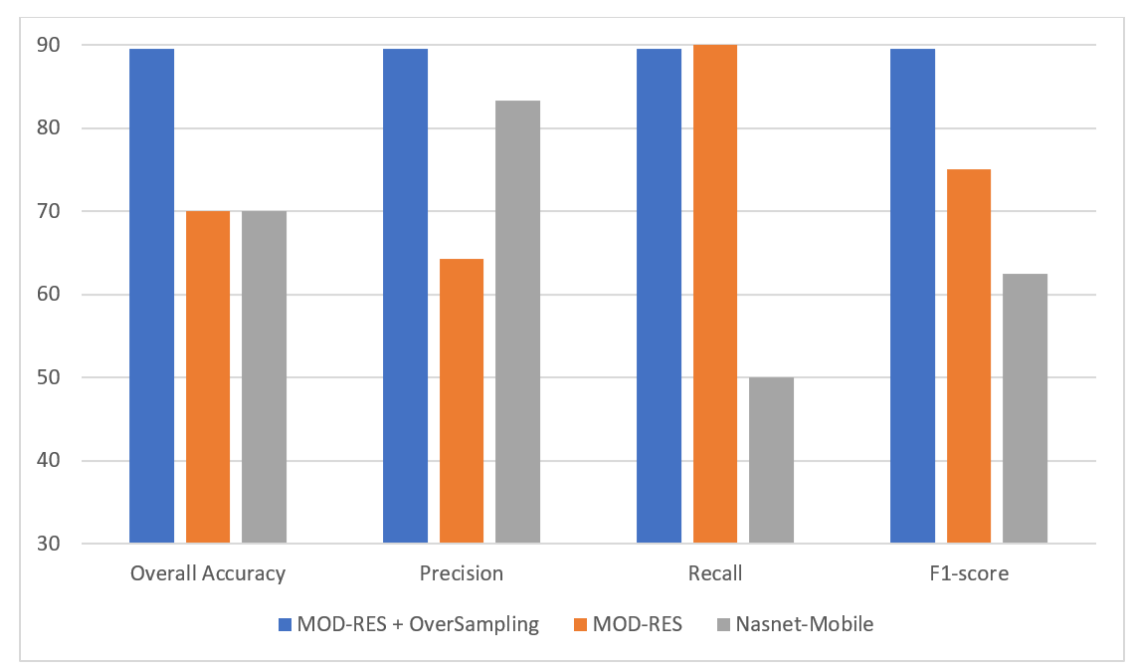

Figure 10. Best result for all models.

Table 8. Comparison of the proposed methodology with state-of-the-art systems.

\begin{tabular}{|c|c|c|c|c|}
\hline Recent Work & Technique & Dataset & Number of Images & Accuracy \\
\hline $\begin{array}{l}\text { Proposed } \\
\text { Methodology }\end{array}$ & MOD-RES & MIAS & $\begin{array}{ll}\text { - } & 51 \text { malignant } \\
\text { - } \quad 48 \text { benign }\end{array}$ & $89.5 \%$ \\
\hline Charan et al. [23] & $\mathrm{CNN}$ & MIAS & $\begin{array}{l}189 \text { normal } \\
133 \text { abnormal }\end{array}$ & $65 \%$ \\
\hline Z. Hussain et al. [15] & VGG-16 & DDSM & $\begin{array}{l}1650 \text { mass } \\
1651 \text { non-mass } \\
\text { (normal) }\end{array}$ & $88 \%$ \\
\hline L. Falconi et al. [9] & VGG & CBIS-DDSM & $\begin{array}{ll}\text { - } & 912 \text { Benign } \\
\text { - } & 784 \text { Malignant }\end{array}$ & $84.4 \%$ \\
\hline S. Eldin et al. [31] & DenseNet-169 & $\mathrm{BACH}$ & $\begin{array}{ll}\text { - } & 100 \text { normal } \\
\text { - } & 100 \text { benign } \\
\text { - } & 100 \text { in-situ } \\
\text { carcinomas } \\
\text { - } & 100 \text { invasive } \\
\text { carcinoma }\end{array}$ & $82 \%$ \\
\hline S. Eldin et al. [31] & ResNet50 & $\mathrm{BACH}$ & $\begin{array}{ll}\text { - } & 100 \text { normal } \\
\text { - } & 100 \text { benign } \\
\text { - } & 100 \text { in-situ } \\
\text { - } & \text { carcinomas } \\
100 \text { invasive } \\
\text { carcinoma }\end{array}$ & $85 \%$ \\
\hline S. Eldin et al. [31] & ResNet101 & $\mathrm{BACH}$ & $\begin{array}{ll}- & 100 \text { normal } \\
- & 100 \text { benign } \\
- & 100 \text { in-situ } \\
\text { carcinomas } \\
\text { - } & 100 \text { invasive } \\
\text { carcinoma }\end{array}$ & $88 \%$ \\
\hline
\end{tabular}


Table 8. Cont.

\begin{tabular}{|c|c|c|c|c|}
\hline Recent Work & Technique & Dataset & Number of Images & Accuracy \\
\hline S. Siddeeq et al. [32] & ResNet & INbreast & 115 total images & $85.9 \%$ \\
\hline K. Shaikh [19] & $\mathrm{CNN}$ & $\begin{array}{l}\text { MIAS, DDSM, } \\
\text { and BancoWeb } \\
\text { LAPIMO }\end{array}$ & $\begin{array}{ll}- & 211 \text { normal } \\
- & 110 \text { cancerous }\end{array}$ & $87.5 \%$ \\
\hline $\begin{array}{l}\text { S. Salvi and } \\
\text { A. Kadam, [33] }\end{array}$ & $\mathrm{CNN}$ & Private Dataset & $\begin{array}{l}178,059 \text { normal } \\
70,132 \text { cancerous }\end{array}$ & $87.84 \%$ \\
\hline W. Sun et al. [34] & $\begin{array}{l}\text { CNN with } \\
\text { Semi-Supervised } \\
\text { Learning (SSL) } \\
\text { algorithm }\end{array}$ & $\begin{array}{l}\text { full-field digital } \\
\text { mammography } \\
\text { (FFDM) }\end{array}$ & $\begin{array}{l}3158 \text { region of } \\
\text { interests (ROI) }\end{array}$ & $82.43 \%$ \\
\hline Roy et al. [35] & $\mathrm{CNN}$ & ICIAR 2018 & $\begin{array}{ll}- & 100 \text { normal } \\
- & 100 \text { benign } \\
- & 100 \text { in-situ } \\
\text { - } & \text { carcinomas } \\
100 \text { invasive } \\
\text { carcinoma }\end{array}$ & $87.4 \%$ \\
\hline S. Alanazi et al. [36] & $\mathrm{CNN}$ & $\begin{array}{c}\text { Kaggle } 162 \mathrm{H} \\
\text { and } \mathrm{E}\end{array}$ & $\begin{array}{l}277,524 \text { total } \\
\text { images }\end{array}$ & $87 \%$ \\
\hline S. Singh et al. [37] & $\begin{array}{c}\text { Histogram } \\
\text { matching }(\mathrm{HM}) \\
\text { and DL } \\
\text { fine-tuning }\end{array}$ & FFDM & $\begin{array}{l}830,450 \text { total } \\
\text { images }\end{array}$ & $84.7 \%$ \\
\hline K. Mendel et al. [38] & CNN and SVM & FFDM & 78 total images & $89 \%$ \\
\hline $\begin{array}{c}\text { A. Rodriguez-Ruiz } \\
\text { et al. [39] }\end{array}$ & $\mathrm{CNN}$ & Private Dataset & 100 total images & $88 \%$ \\
\hline M. Yousefi et al. [40] & $\mathrm{CCN}$ & $\begin{array}{c}\text { Research } \\
\text { Laboratory at } \\
\text { Massachusetts } \\
\text { General Hospital } \\
\text { (MGH) }\end{array}$ & 5040 total images & $87 \%$ \\
\hline
\end{tabular}

\section{Conclusions}

Two reliable and automatic mechanisms for breast cancer diagnosis are presented using mammographic images to differentiate between benign and malignant infected subjects. To improve the intensity of the mammographic image and eliminate any noise, the suggested system employs image enhancement techniques. Two alternative DL approaches, Nasnet-Mobile and MOD-RES were trained on top of preprocessed mammographic images to avoid overfitting and increase the overall capabilities of the proposed DL systems. A mammographic image dataset called the MIAS dataset was utilized to assess the proposed system's effectiveness. The suggested method outperforms professional radiologists with an overall accuracy of $89.5 \%$, precision of $89.5 \%$, recall of $89.5 \%$, and F1-score of $89.5 \%$ using MOD-RES + oversampling, while the overall accuracy reaches $70 \%$, precision of $83.3 \%$, recall of $50 \%$, and an F1-score of $62.5 \%$ using Nasnet-Mobile. According to comparative studies, the proposed system (MOD-RES + oversampling) beats existing models.

Author Contributions: Conceptualization, M.A. and W.G.; methodology, M.A. and W.G.; software, M.A. and W.G.; validation, M.A. and W.G.; formal analysis, M.A. and W.G.; investigation, M.A. and W.G.; resources M.A. and W.G.; data curation, M.A. and W.G.; writing-original draft preparation, M.A. and W.G.; writing-review and editing, M.A. and W.G.; visualization, M.A. and W.G.; supervision, M.A. and W.G.; project administration, M.A. and W.G.; funding acquisition, M.A. and W.G. All authors have read and agreed to the published version of the manuscript.

Funding: This research was funded by JOUF UNIVERSITY, grant number (DSR-2021-02-0356). 


\section{Institutional Review Board Statement: Not applicable.}

Informed Consent Statement: Not applicable.

Data Availability Statement: http:/ / peipa.essex.ac.uk/info/mias.html, accessed on 29 November 2021.

Acknowledgments: The authors extend their appreciation to the Deanship of Scientific Research at Jouf University for funding this work through Research Grant No (DSR-2021-02-0356).

Conflicts of Interest: The authors declare that they have no conflict of interest to report regarding the present study.

\section{References}

1. Macías-García, L.; Martínez-Ballesteros, M.; Luna-Romera, J.M.; García-Heredia, J.M.; García-Gutiérrez, J.; Riquelme-Santos, J.C. Autoencoded DNA methylation data to predict breast cancer recurrence: Machine learning models and gene-weight significance. Artif. Intell. Med. 2020, 110, 101976. [CrossRef]

2. Yap, M.H.; Goyal, M.; Osman, F.; Martí, R.; Denton, E.; Juette, A.; Zwiggelaar, R. Breast ultrasound region of interest detection and lesion localisation. Artif. Intell. Med. 2020, 107, 101880. [CrossRef]

3. Gouda, W.; Selim, M.M.; Elshishtawy, T. An Approach for Breast Cancer Mass Detection in Mammograms. 2012. Available online: https://www.bu.edu.eg/portal/uploads/Engineering,\%20Shoubra/Electrical\%20Engineering/839/publications/ Walaa $\% 20$ Gouda\%20Hassan\%20Mohammed_An\%20Approach $\% 20$ for $\% 20$ Breast $\% 20$ Cancer $\% 20$ Mass $\% 20$ Detection $\% 20$ in $\% 20$ Mammograms.pdf (accessed on 18 January 2022).

4. Mehmood, M.; Ayub, E.; Ahmad, F.; Alruwaili, M.; Alrowaili, Z.A.; Alanazi, S.; Rizwan, M.H.M.; Naseem, S.; Alyas, T. Machine learning enabled early detection of breast cancer by structural analysis of mammograms. Comput. Mater. Contin. 2021, 67, 641-657. [CrossRef]

5. Rahman, A.S.A.; Belhaouari, S.B.; Bouzerdoum, A.; Baali, H.; Alam, T.; Eldaraa, A.M. Breast Mass Tumor Classification using Deep Learning. In Proceedings of the 2020 IEEE International Conference on Informatics, IoT, and Enabling Technologies (ICIoT), Doha, Qatar, 2-5 February 2020; IEEE: New York, NY, USA, 2020.

6. Wang, H.; Li, Y.; A Khan, S.; Luo, Y. Prediction of breast cancer distant recurrence using natural language processing and knowledge-guided convolutional neural network. Artif. Intell. Med. 2020, 110, 101977. [CrossRef]

7. Rao, A.P.; Bokde, N.; Sinha, S. Photoacoustic imaging for management of breast cancer: A literature review and future perspectives. Appl. Sci. 2020, 10, 767. [CrossRef]

8. Sinha, N. SSEGEP: Small SEGment Emphasized Performance evaluation metric for medical image segmentation. arXiv 2021, arXiv:2109.03435.

9. Falconi, L.G.; Perez, M.; Aguila, W.G.; Conci, A. Transfer learning and fine tuning in breast mammogram abnormalities classification on CBIS-DDSM database. Adv. Sci. Technol. Eng. Syst. J. 2020, 5, 154-165. [CrossRef]

10. Alkhaleefah, M.; Ma, S.-C.; Chang, Y.-L.; Huang, B.; Chittem, P.K.; Achhannagari, V.P. Double-shot transfer learning for breast cancer classification from X-ray images. Appl. Sci. 2020, 10, 3999. [CrossRef]

11. Chatfield, K.; Simonyan, K.; Vedaldi, A.; Zisserman, A. Return of the devil in the details: Delving deep into convolutional nets. arXiv 2014, arXiv:1405.3531.

12. Simonyan, K.; Zisserman, A. Very deep convolutional networks for large-scale image recognition. arXiv 2014, arXiv:1409.1556.

13. Shen, D.; Wu, G.; Suk, H.-I. Deep learning in medical image analysis. Med. Image Anal. 2017, 19, 221-248. [CrossRef] [PubMed]

14. Litjens, G.; Kooi, T.; Bejnordi, B.E.; Setio, A.A.A.; Ciompi, F.; Ghafoorian, M.; van der Laak, J.A.; van Ginneken, B.; Sánchez, C.I. A survey on deep learning in medical image analysis. Med. Image Anal. 2017, 42, 60-88. [CrossRef] [PubMed]

15. Hussain, Z.; Gimenez, F.; Yi, D.; Rubin, D. Differential data augmentation techniques for medical imaging classification tasks. AMIA Annu. Symp. Proc. Arch. 2017, 2017, 979-984.

16. Perre, A.C.; Alexandre, L.; Freire, L.C. Lesion classification in mammograms using convolutional neural networks and transfer learning. Comput. Methods Biomech. Biomed. Eng. Imaging Vis. 2018, 7, 550-556. [CrossRef]

17. Khamparia, A.; Bharati, S.; Podder, P.; Gupta, D.; Khanna, A.; Phung, T.K.; Thanh, D.N.H. Diagnosis of breast cancer based on modern mammography using hybrid transfer learning. Multidimens. Syst. Signal Process. 2021, 32, 747-765. [CrossRef]

18. Kaur, P.; Singh, G.; Kaur, P. Intellectual detection and validation of automated mammogram breast cancer images by multi-class SVM using deep learning classification. Inform. Med. Unlocked 2019, 16, 100151. [CrossRef]

19. Shaikh, K.; Krishnan, S.; Thanki, R. Deep Learning Model for Classification of Breast Cancer, in Artificial Intelligence in Breast Cancer Early Detection and Diagnosis; Springer: Berlin/Heidelberg, Germany, 2021; pp. 93-100.

20. Wahab, N.; Khan, A.; Lee, Y.S. Transfer learning based deep CNN for segmentation and detection of mitoses in breast cancer histopathological images. Microscopy 2019, 68, 216-233. [CrossRef]

21. Jiang, F.; Liu, H.; Yu, S.; Xie, Y. Breast mass lesion classification in mammograms by transfer learning. In Proceedings of the 5th International Conference on Bioinformatics and Computational Biology, Hong Kong, China, 6-8 January 2017.

22. Cao, H.; Bernard, S.; Heutte, L.; Sabourin, R. Improve the performance of transfer learning without fine-tuning using dissimilaritybased multi-view learning for breast cancer histology images. In Proceedings of the 15th International Conference, ICIAR 2018, Póvoa de Varzim, Portugal, 27-29 June 2018; Springer: Berlin/Heidelberg, Germany, 2018. 
23. Charan, S.; Khan, M.J.; Khurshid, K. Breast cancer detection in mammograms using convolutional neural network. In Proceedings of the 2018 International Conference on Computing, Mathematics and Engineering Technologies (iCoMET), Wuhan, China, 7-8 February 2018; IEEE: New York, NY, USA, 2018.

24. Suckling, J.P. The mammographic image analysis society digital mammogram database. Digil. Mammo 1994, 375-386.

25. Russakovsky, O.; Su, H.; Krause, J.; Satheesh, S.; Ma, S.; Huang, Z.; Karpathy, A.; Khosla, A.; Bernstein, M.; Berg, A.C.; et al. Imagenet large scale visual recognition challenge. Int. J. Comput. Vis. 2015, 115, 211-252. [CrossRef]

26. Saxen, F.; Werner, P.; Handrich, S.; Othman, E.; Dinges, L.; Al-Hamadi, A. Face attribute detection with mobilenetv2 and nasnet-mobile. In Proceedings of the 2019 11th International Symposium on Image and Signal Processing and Analysis (ISPA), Dubrovnik, Croatia, 23-25 September 2019; IEEE: New York, NY, USA, 2019.

27. Reddy, N.; Rattani, A.; Derakhshani, R. Comparison of deep learning models for biometric-based mobile user authentication. In Proceedings of the 2018 IEEE 9th International Conference on Biometrics Theory, Applications and Systems (BTAS), Redondo Beach, CA, USA, 22-25 October 2018; IEEE: New York, NY, USA, 2018.

28. He, K.; Zhang, X.; Ren, S.; Sun, J. Deep residual learning for image recognition. In Proceedings of the IEEE Conference on Computer Vision and Pattern Recognition (CVPR), Las Vegas, NV, USA, 26 June-1 July 2016.

29. Basha, S.S.; Dubey, S.R.; Pulabaigari, V.; Mukherjee, S. Impact of fully connected layers on performance of convolutional neural networks for image classification. Neurocomputing 2020, 378, 112-119. [CrossRef]

30. Renard, F.; Guedria, S.; De Palma, N.; Vuillerme, N. Variability and reproducibility in deep learning for medical image segmentation. Sci. Rep. 2020, 10, 13724. [CrossRef] [PubMed]

31. Eldin, S.N.; Hamdy, J.K.; Adnan, G.T.; Hossam, M.; Elmasry, N.; Mohammed, A. Deep Learning Approach for Breast Cancer Diagnosis from Microscopy Biopsy Images. In Proceedings of the 2021 International Mobile, Intelligent, and Ubiquitous Computing Conference (MIUCC), Cairo, Egypt, 26-27 May 2021; IEEE: New York, NY, USA, 2021.

32. Siddeeq, S.; Li, J.; Ali Bhatti, H.M.; Manzoor, A.; Malhi, U.S. Deep Learning RN-BCNN Model for Breast Cancer BI-RADS Classification. In Proceedings of the 2021 The 4th International Conference on Image and Graphics Processing, Sanya, China, 1-3 January 2021.

33. Salvi, S.; Kadam, A. Breast Cancer Detection Using Deep learning and IoT Technologies. J. Phys. Conf. Ser. 2021, 1831, 012030. [CrossRef]

34. Sun, W.; Tseng, T.-L.B.; Zhang, J.; Qian, W. Enhancing deep convolutional neural network scheme for breast cancer diagnosis with unlabeled data. Comput. Med. Imaging Graph. 2017, 57, 4-9. [CrossRef] [PubMed]

35. Roy, K.; Banik, D.; Bhattacharjee, D.; Nasipuri, M. Patch-based system for classification of breast histology images using deep learning. Comput. Med. Imaging Graph. 2019, 71, 90-103. [CrossRef] [PubMed]

36. Alanazi, S.A.; Kamruzzaman, M.M.; Sarker, N.I.; Alruwaili, M.; Alhwaiti, Y.; Alshammari, N.; Siddiqi, M.H. Boosting breast cancer detection using convolutional neural network. J. Healthc. Eng. 2021, 2021, 5528622. [CrossRef]

37. Singh, S.; Matthews, T.P.; Shah, M.; Mombourquette, B.; Tsue, T.; Long, A.; Almohsen, R.; Pedemonte, S.; Su, J. Adaptation of a deep learning malignancy model from full-field digital mammography to digital breast tomosynthesis. In Proceedings of the Medical Imaging 2020: Computer-Aided Diagnosis, Houston, TX, USA, 15-20 February 2020; International Society for Optics and Photonics: Bellingham, WA, USA, 2020.

38. Mendel, K.; Li, H.; Sheth, D.; Giger, M. Transfer learning from convolutional neural networks for computer-aided diagnosis: A comparison of digital breast tomosynthesis and full-field digital mammography. Acad. Radiol. 2019, 26, 735-743. [CrossRef]

39. Rodriguez-Ruiz, A.; Teuwen, J.; Vreemann, S.; Bouwman, R.W.; E Van Engen, R.; Karssemeijer, N.; Mann, R.; Gubern-Merida, A.; Sechopoulos, I. New reconstruction algorithm for digital breast tomosynthesis: Better image quality for humans and computers. Acta Radiol. 2018, 59, 1051-1059. [CrossRef]

40. Yousefi, M.; Krzyżak, A.; Suen, C.Y. Mass detection in digital breast tomosynthesis data using convolutional neural networks and multiple instance learning. Comput. Biol. Med. 2018, 96, 283-293. [CrossRef] 